\title{
Methods of Visualisation for Flows with Internal Waves Attractors
}

\author{
D.A Ryazanov ${ }^{1, A}$, S.A. Elistratov²,B, M.V. Kraposhin3,A \\ A Ivannikov Institute for System Programming of the RAS \\ ${ }^{B}$ Lomonosov Moscow State University \\ 1 ORCID: 00oo-0001-9568-7121, psuburner@gmail.com \\ 2 ORCID: 0000-0002-7006-6879 \\ 3 ORCID: 0000-0001-5730-2702, os-cfd@yandex.ru
}

\begin{abstract}
Application of different approaches for visualization of hydrodynamic fields of internal waves is closely connected with the possibility of extracting important numerical characteristics of the flows. In this paper we describe methods of visualisation, which were shown to be very effective for the illustration of the internal wave attractors both for laboratory experiments and numerical simulations. On the other hand, some novel approaches for description of the wave flows with accumulation of energy are discussed. Methods of vortices identification show interesting properties of the wave flows, and may give an alternative for the estimation of the width of wave beams. At the same time, the limitations of the applicability of the described methods strongly depend on the space and time resolution of the available data, which is especially important in the areas of the internal wave beam reflection.
\end{abstract}

Keywords: CFD, internal waves, inertial waves, post-processing, wave attractor, opensource software.

\section{Introduction}

Internal waves are may be the most common types of waves in oceans, since even if the ocean surface seems to be calm, the deep-ocean waves $[1,2]$ are always present ${ }^{1}$. Oceanologists, biologists, ecologists, and technician have specific interests in the study of internal waves. This phenomenon is partly responsible for the vertical mixing of the stratified fluids, the migration of living organisms, the redistribution of energy in the ocean, and the propagation of various kinds of impurities and pollution.

Despite a great diversity of experimental approaches for visualisation of weakly compressible flows [4,5], the experiments are often bounded by the possibility to assess some interesting propriety at certain time and location. Concerning the internal attractors and accompanying phenomena, remarkable advantages have been achieved since 1990s. Besides the successful application of traditional approaches based on synthetic schlieren, particle image velocimetry (PIV) and planar laser-induced fluorescence (PLIF), some methods based on signal analysis, which traditionally were more applied to electromagnetic fields, showed remarkable relevance for description of the internal or inertial wave dynamics [6-9]. Meanwhile, the time and space resolution of these methods is limited, and sometimes the laboratory data do not allow to reconstruct the hydrodynamic fields, as mentioned in [10] concerning the reconstruction of the stream function based on laboratory data near the boundaries.

\footnotetext{
1 "Gravity waves in the ocean's interior are as common as waves at the sea surface - perhaps even more so, for no one has ever reported an interior calm” [3]
} 
Internal wave appears as a result of perturbations of stably stratified fluid. One of the major sources of the external forcing, resulting in internal waves, is the tidal effect produced by the orbital motions of the Moon and the Sun. These global flows interact with the ocean bottom irregularities and generate internal waves. The very special feature of the internal waves is that in case of constant stratification the angle of the wave beams and the law of reflection are defined by the dispersion relation:

$$
\frac{\omega_{0}}{N}=\sin (\theta)
$$

where $\omega_{0}$ is the forcing frequency determined by external forcing on the fluid; $N=$ $\sqrt{-\frac{g}{\rho(z)} \cdot \frac{d \rho(z)}{d z}}$ buoyancy frequency which determine distribution of density $(\rho(z))$.

Due to these properties internal waves can be focused upon reflection from the oblique wall. As a consequence, the internal wave wavelength is reduced upon reflection but its amplitude increases.

The great interest is the result of the consecutive reflections. It can be obtained by the monochromatic excitation of the internal waves in the closed domain with the oblique boundaries, the simplest example of which is the trapeze with one inclined boundary. A remarkable property of the billiard in trapezoid geometry is that focusing prevails over defocusing. If the internal waves beams propagate in trapezoid tank, focusing occurs continuously and all the wave beams converge to closed trajectory. The simplest one is a parallelogram with four reflection points located at the sides of the trapeze (Fig. 1a).

For the first time the phenomenon of wave attractors was described by Leo Maas $[11,12]$ who studied the convergence of wave beams in different geometries.

First attempts of numerical study [13] of internal wave attractors successfully reproduced qualitatively the 2D structure of internal wave attractors in trapezoidal domains, though quantitatively the velocity amplitude was too high as compared with the experiment. $3 \mathrm{D}$ numerical simulations $[14,15]$ resolved this discrepancy through estimation of dissipation and boundary layers effects.

In this paper we apply the before-mentioned methods for the results of numerical experiments, and discuss the methods, which were not yet widely used for analysis of internal wave attractors.

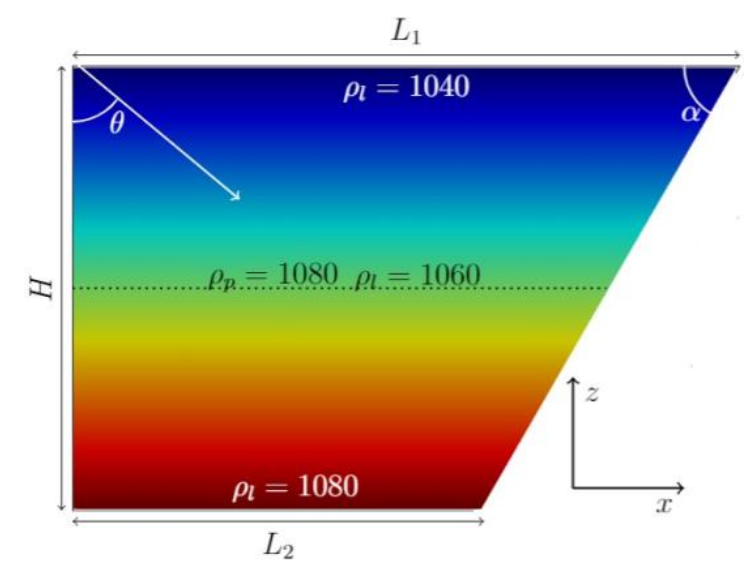

(a) Initial distribution of density. $\rho_{l}-$ density of liquid, $\rho_{p}$ - density of particles, $\theta=$ $\arcsin \left(\frac{\omega}{N}\right)$, where $\omega$ is frequency of wavemaker and $N$ is the buoyancy frequency.

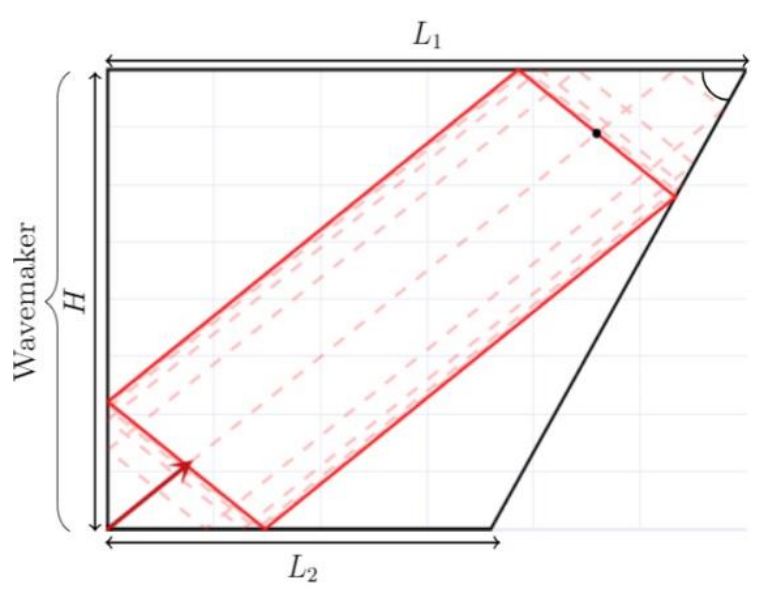

(b) Ray-tracing of an internal wave beam subject to the dispersion relation (1).

Figure 1: Scheme of computational domain for 2D attractor of internal waves. Initial distribution of density (a), and shape of attractor in ideal fluid (b). 


\section{Numerical setup}

Laboratory and numerical study of linear and nonlinear dynamics of wave attractors in three-dimensional setup showed a good agreement in terms of major hydrodynamic properties both in laminar and turbulent regimes [14, 15]. High order spectral element approach, which was used in these simulations is still hard to adopt for multi-phase flows. In this study we consider the processes of sedimentation, and numerical simulation with the help of quasihydrodynamic approach $[16,17]$.

Equations for mass, momentum, and salinity transport and diffusion in quasihydrodynamic approach can be written in the following form [16]:

$$
\begin{gathered}
\nabla \cdot(\vec{U}-\vec{W})=0, \\
\frac{\partial \vec{U}}{\partial t}+\nabla \cdot((\vec{U}-\vec{W}) \otimes \vec{U})-\nabla \cdot v\left(\nabla \vec{U}+(\nabla \vec{U})^{T}\right)-\nabla \cdot(\vec{U} \otimes \vec{W})=-\frac{1}{\rho_{m}} \nabla \hat{p}+\vec{F}, \\
\frac{\partial s}{\partial t}+\nabla \cdot((\vec{U}-\vec{W}) s)-\nabla \cdot \frac{v}{S c}(\nabla s)=0,
\end{gathered}
$$

where reduced pressure $\hat{p}=p-p_{0}$, restoring force $\vec{F}=\beta \vec{g} \hat{s}, \vec{U}-$ velocity field.

$\hat{s}=s(x, y, z, t)-s(x, y, z, 0)-\quad$ salinity destribution, $\quad \vec{W}=\tau\left(\vec{U} \cdot \nabla \vec{U}+\frac{1}{\rho_{m}} \nabla \hat{p}-\vec{F}\right)-$ additional velocity according to quasihydrodynamic approach, $v-$ viscosity, $S c-$ Schmidt number.

Geometrically the computational domain is a rectangular trapeze, with the wavemaker located at the left wall, and inclined right wall, as in the experiments $[14 ; 18]$.

Boundary conditions at the fixed walls:

$$
\vec{U}=0, \frac{\partial \tilde{p}}{\partial \vec{n}}=\rho_{0} \vec{n} \cdot\left(-\vec{U}_{b} \cdot \nabla \vec{U}+\vec{F}\right), \frac{\partial s}{\partial \vec{n}}=0 .
$$

The initial conditions for a passive scalar are chosen so that the buoyancy frequency is equal to 1

$$
N(z)=\sqrt{-\frac{g}{\rho_{m}} \cdot \frac{d \rho(z)}{d z}}=1,
$$

where $\rho(z)=\rho_{m}(1+\beta s), \quad \rho_{m}$ is minimal density, and $\beta-$ coefficient of salinity contraction.

Equations (2-4) were approximated with the help of finite volume method and opensource code OpenFOAM v2012 with QHDFoam solver [19]

\section{Post-processing and data analysis}

The results of numerical simulation were approximated at a regular rectangular grid with the help of openFoam functionObject and processed by python scripts. Hydrodynamic fields were visualised with the help of open-source, multi-platform data analysis and visualization platform paraview [20].

Parameters of the simulation are chosen to be close to the corresponding laboratory experiments. As follows from the theoretical predictions and laboratory experiments, it is expected that internal waves energy will concentrate in the zone of attraction (Fig. 1b).

Geometry of the tank filled with salty water is given by: $H=0.4 \mathrm{~m}, L_{1}=0.6 \mathrm{~m}, L_{2}=$ $0.39 \mathrm{~m}$. The buoyancy frequency $N=1$.

Boundary conditions at the left wall with the wavemaker read:

where $a=0.003 \mathrm{~m}, \omega_{0}=0.63 \mathrm{~s}^{-1}$.

$$
U_{x}=a \cdot \cos \left(\frac{\pi \cdot z}{H}\right) \cdot \omega_{0} \cdot \sin \left(\omega_{0} t\right),
$$


In the figures below we will use wavemaker frequency $f_{0}=\frac{\omega_{0}}{2 \pi}$ and corresponding period $T_{0}=f_{0}^{-1}$.

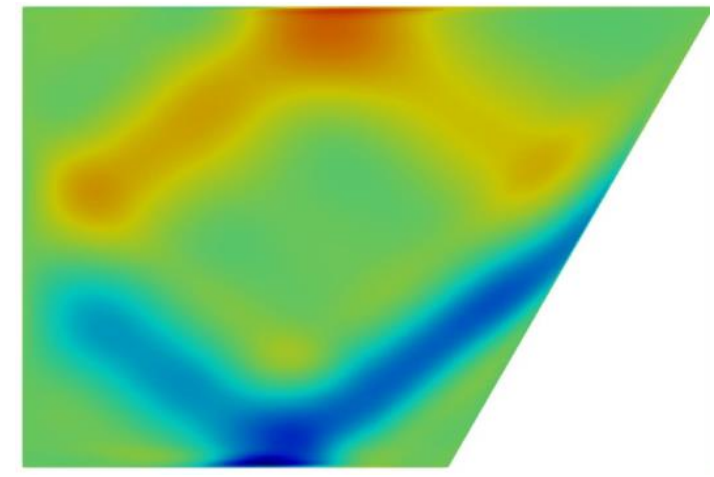

(a) Internal waves attractor formation $t=60 \mathrm{~s}$ to $t=80 \mathrm{~s}$

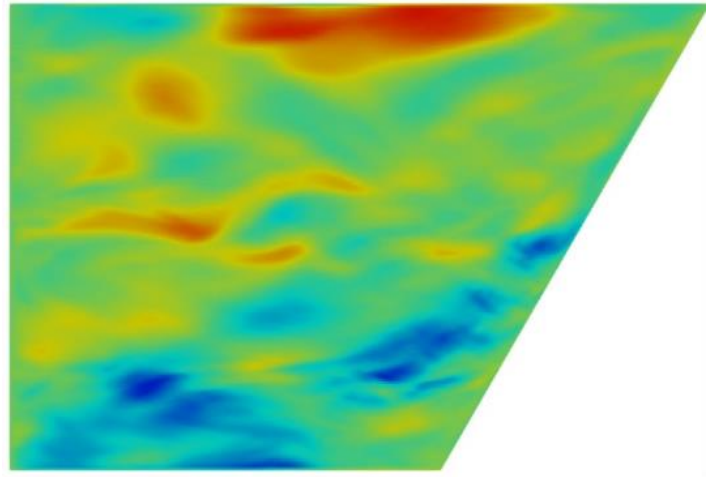

(b) Internal waves turbulence $t=3590 \mathrm{~s}$ to $t=3600 \mathrm{~s}$

Figure 2: Horizontal component of velocity, (a) after formation of internal wave attractor with finite width, (b) instability development and transition to fully turbulent motion.

Visualization of velocity field was provided by open-source software paraview [20]. In figure 2 the horizontal component is shown. About 20 first wavemaker periods attractor is being formed, next the attractor holds the shape during about 30 periods, after 50 periods the perturbation are grown enough to initiate the cascade of the triadic instabilities.

As the one of main method of flow motions analysis time-frequency diagram which shows dynamic of spectrum is considered (Fig. 3). It is defined by the Fourier-transform made with a sliding time window. Hence, a vertical slice of the diagram is the spectrum taken over the neighbourhood of time moment $t$, so one can trace the evolution of the spectrum. At the start of the wavemaker the fluid has one clear frequency, but then the attractor becomes prone to hydrodynamic instabilities and the generation of the cascade of secondary waves.

Figure 3 illustrate dependence of time-frequency diagrams on the forcing amplitude (logscale of amplitude, normalized by maximum value). If the amplitude is not high enough, spectrum almost doesn't evolve and has discrete peaks; with the increase of the amplitude the spectrum combines discrete peaks and continuous background and fluctuates with the time, which indicates the transition to the turbulent regime.

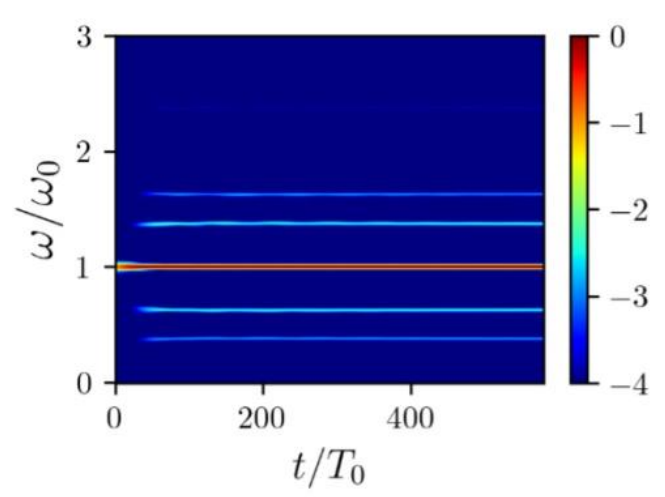

(a) Time-frequency diagram of superharmonics presence $(a=1.4 \mathrm{~mm})$

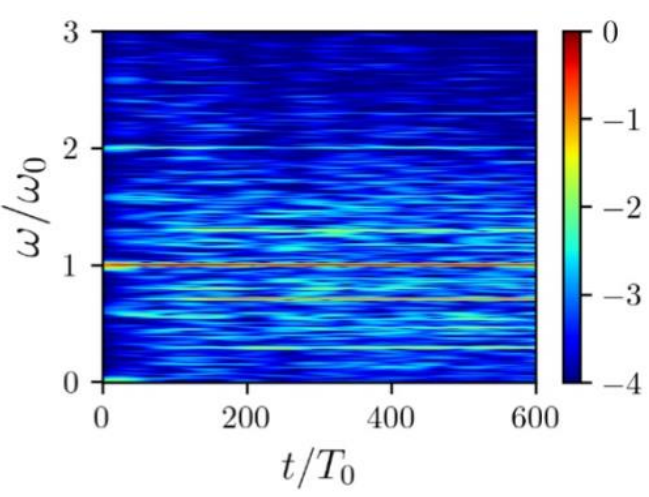

(b) Time-frequency diagram of attractor destruction $(a=3.0 \mathrm{~mm})$

Figure 3: Time-frequency diagrams 


\subsection{Sedimentation}

One of the important problems with applications in oceanology is the sedimentation of suspended particles and their interaction with the internal waves. To describe qualitatively the influence of wave attractors on sedimentation of suspended particles we have made visualization for laminar and turbulent regimes of wave attractors. Initial density profile in the tank is linear. At the initial state spherical particles with diameter $d=0.001 \mathrm{~m}$ were distributed uniformly over the horizontal middle line (Fig. 1a). The model does not account for feedback from the particles on the flow. Such a setup showed drastic differences in the sedimentation properties for laminar and turbulent wave attractors.

Two questions arise: a) redistribution of the particles in the bulk flow before the particles approach the bottom, b) redistribution of the particles near the bottom.

Numerical experiments were carried out for two different regimes, corresponding to wavemaker (7) amplitudes $a_{1}=0.0005 \mathrm{~m}$ - regime of stable attractor without wave instabilities, and $a_{2}=0.003 \mathrm{~m}$ - fully turbulent regime.

Numerical experiment has shown that particles experience oscillation while falling, and in laminar regime they continue to oscillate near the bottom along horizontal direction (Fig. 4). First part of the experiment shows movement of particles until they touch the bottom. Internal waves make weak oscillation of "particles line" (Fig. 4a). And even attractor of internal waves does not affect to particle distribution due to small amplitude (Fig. 4b). For the turbulent regime the long-time behavior of the particles near the bottom is completely different: all the particles are attracted by two points at the bottom during the period $1000-$ $2000 s$ (Fig. 5). At the initial phase of sedimentation (Fig. 5a) internal waves focusing produce intense movement of particles around the bottom of the tank, which leads to a redistribution of particles around two points (Fig. 5b).

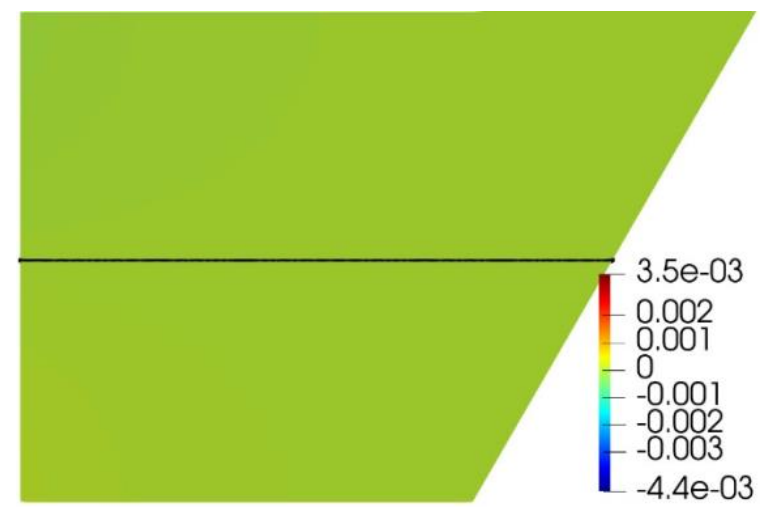

(a) Sedimentation from $t=0 \mathrm{~s}$ to $t=80 \mathrm{~s}$. Initial phase, particles slowly moving to the bottom.

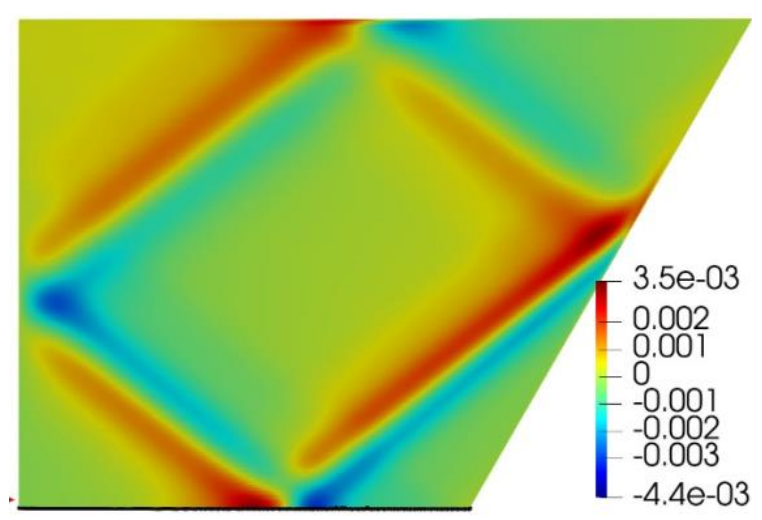

(b) Redistribution from $t=3590 \mathrm{~s}$ to $t=3600 \mathrm{~s}$. Ending stage, particles lie evenly at the bottom.

Figure 4: Sedimentation in presence of stable ("laminar") wave attractor. 


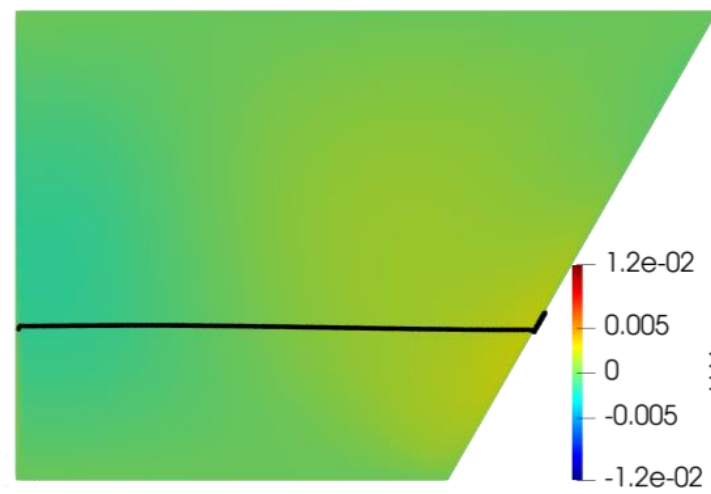

(a) Sedimentation from $t=0 \mathrm{~s}$ to $t=80 \mathrm{~s}$. Initial phase, particles slowly moving to the bottom.

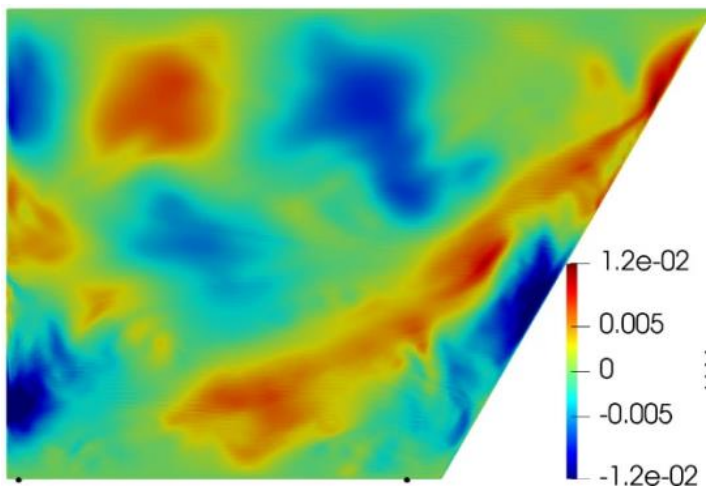

(b) Redistribution from $t=3590 \mathrm{~s}$ to $t=3600 \mathrm{~s}$. Ending stage, particles concentrate around two points at the bottom.

Figure 5: Sedimentation in presence of turbulent wave attractor.

\subsection{Three-dimensional wavemaker}

Three-dimensional setup may result in additional focusing along the transverse direction [21]. Despite the fact that attractor may be located at only part of the left wall, the visualisation of numerical simulation in figure 7 shows almost perfect two-dimensional structure.

With addition third dimension the simulation problem was complicated significantly. There are difficulties in visualizing a three-dimensional velocity field. For showing destruction of internal waves attractor paraview filter 'threshold' was used. It allows to see values from gap and detect areas with intensive motion (Fig. 7). Emptiness is proposed to fill in salinity field with interprocess patches (glares). And finally, with 'slice' filter select closest to wavemaker area for visualisation of wavemaker motion.

Visual analysis of simulation shows internal waves attractors destruction procedure. The attractor is formed in the period from 0 to 10 oscillation periods, then from 10 to 30 oscillation periods stable and attractor structure is observed and finally from 30 to 300 and belong difference of velocities become too big that waves overturn and incoming energy produce secondary waves.

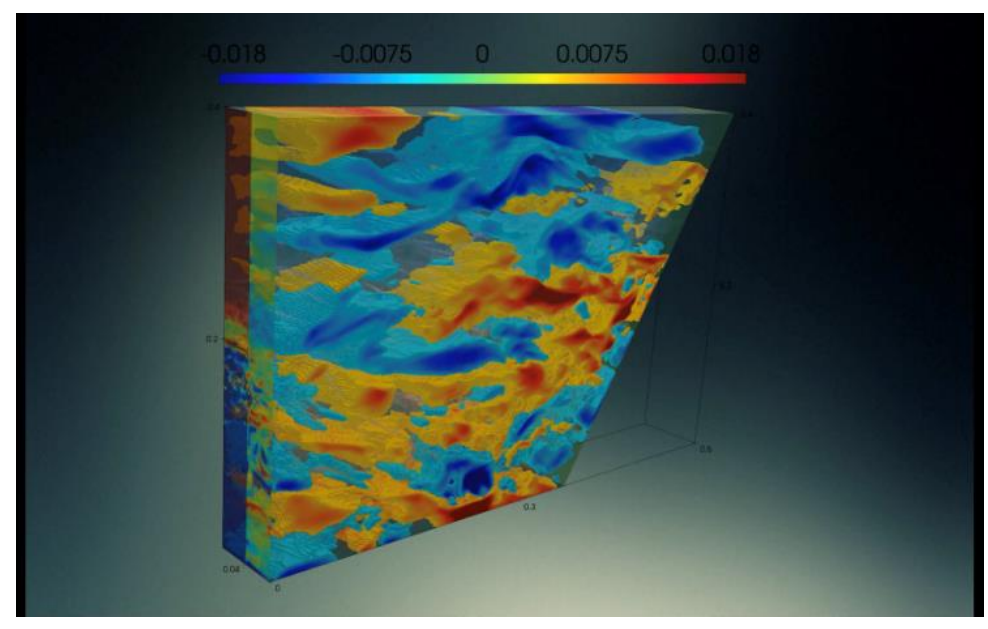

Figure 7: Life cycle of internal waves attractor from stability to chaos. Color visualization of velocity horizontal component. Flare in reservoir is an interprocessors interfaces painted in salinity colorbar where 1 is a blue and 2 is a red. Wall with wave maker has a different color scale: from $-0.001 \mathrm{~m} / \mathrm{s}$ to $\$ 0.001 \mathrm{~m} / \mathrm{s}$. 


\section{Conclusions}

As a result of direct numerical simulation of internal wave turbulence, one gets a large volume of data. It is now possible to get high-resolution data, and resolve the small-scale flows. At the same time, it requires more computational resources to generate and process it. Raw data is difficult to interpret without special software. In this paper we consider some open-source software for flow analysis and visualization. The open-source software complies with the principles of scientific credibility, ability to modify and reuse the code, and source code transparency.

To obtain hydrodynamic fields OpenFOAM was used, but raw data takes up a lot of space on hard drives and generate a lot of files especially in simulations with particles. Sometimes it can be critical. At this paper functionObject 'vtkWrite' was used to resolve this problem. For simulation with particles 'cloudWrite' function object was used which generate much fewer files then raw pure OpenFOAM output. Additionally, data was collected at key nodes for Fourier analysis and construction of time-frequency diagrams with 'postProcWrite' functionObject.

Problem of internal gravity waves attractor simulation is complicated by the fact that it is required to simulate significant periods of model time. 24 processors for $300 \times 200$ computational domain needs for simulation of 2D problem. Simulation of a 2D problem lasted for 5 days on Unihub cluster [22]. 3D problem required more detailed mesh additionally to the 3 -d dimension. 196 processors for $375 \times 250 \times 16$ domain. Simulation of 450 seconds lasted for 7 days on The Lobachevsky supercomputer.

Processing of simulation results required many different instruments for calculations and visualisation. Fourier analysis was provided with python package scipy [23], and drawing with matplotlib [24]. Visualisation was obtained by paraview [20]. Scripts for vortices visualisation was developed with python [25].

\section{Acknowledgments}

The research was supported by RSF (project No. 19-11-00169). The authors thank Elizarova T. G. for continuous support.

\section{References}

1. Munk, W. Abyssal recipes // Deep-Sea Res. - 1966. - Vol. 13. - P. 707-730.

2. W. Munk, C. Wunsch // Deep Sea Research Part I: Oceanographic Research Papers. 1998. - Vol. 45. - Abyssal recipes II. - № 12. - P. 1977-2010.

3. Munk, W.H. 9 internal waves and small-scale processes -2005 .

4. Znamenskaya, I.A. Methods for panoramic visualization and digital analysis of thermophysical flow fields // Scientific Visualization. - 2021. - Vol. 13. - № 3. - P. 125-158.

5. B. Sutherland, T. Dauxois, T. Peacock // Modeling atmospheric and oceanic flows. American Geophysical Union (AGU), 2014. - P. 193-212.

6. Bourget, $B$. Experimental study of parametric subharmonic instability for internal plane waves - 2013. - Vol. 723. - P. 1-20.

7. Dossmann, $Y$. Mixing by internal waves quantified using combined PIV/PLIF technique // Experiments in Fluids. - 2016. - Vol. 57. - № 8. - P. 132.

8. Davis, G. Energy budget in internal wave attractor experiments // Journal of Fluid Mechanics. - 2019. - Vol. 880. - P. 743-763.

9. Husseini, $P$. Experimental study on superharmonic wave generation by resonant interaction between internal wave modes // Phys. Rev. Fluids. - 2020. - Vol. 5. - P. 074804.

10. Lee, F.M. Experimental determination of radiated internal wave power without pressure field data // Physics of Fluids. - 2014. - Vol. 26. - № 4. - P. 046606.

11. Maas, L.R.M. Geometric focusing of internal waves // Journal of Fluid Mechanics. 1995. - Vol. 300. - P. 1-41. 
12. Maas, L.R.M. Observation of an internal wave attractor in a confined, stably stratified fluid // Nature. - 1997. - Vol. 388. - P. 557-561.

13. Grisouard, N., C. Staquet, I. Pairaud, Numerical simulation of a two-dimensional internal wave attractor // Journal of Fluid Mechanics. - 2008. - Vol. 614.

14. Brouzet, $C$. Internal wave attractors examined using laboratory experiments and $3 \mathrm{D}$ numerical simulations // Journal of Fluid Mechanics. - 2016. - Vol. 793. - P. 109-131.

15. Brouzet, $C$. Energy cascade in internal-wave attractors // EPL (Europhysics Letters). - 2016. - Vol. 113. - № 4 .

16. Elizarova, T.G. Quasi-gas dynamic equations - Springer, 2009.

17. Sheretov, Y.V. Continuum dynamics under spatiotemporal averaging // SPC Regular; Chaotic Dynamics. (Moscow-Izhevsk, 2009, in Russian), 2009.

18. Scolan, H., E. Ermanyuk, T. Dauxois Nonlinear Fate of Internal Wave Attractors // Physical Review Letters. - 2013. - Vol. 110. - № 23. - P.

19. OpenFOAM framework for simulation of fluid flows using regularized (QGD/QHD) equations approach. - https://github.com/unicfdlab/QGDsolver, 2019

20. Open-source, multi-platform data analysis and visualization application. https://https://www.paraview.org/, 2000.20.

21. Pillet, $G$. Internal wave attractors in three-dimensional geometries: trapping by oblique reflection // Journal of Fluid Mechanics. - 2018. - Vol. 845. - P. 203-225.

22. Samovarov, O.I., S.S. Gaysaryan. The web-laboratory architecture based on the cloud and the UniHUB implementation as an extension of the OpenStack platform - 2014. Vol. 26. - № 1. - P. 403-420.

23. scipy official website. - https://www.scipy.org/.

24. matplotlib official github. - https://github.com/matplotlib/matplotlib.

25. python official website. - https://www.python.org/. 